\title{
Three-dimensional finite element analysis of optimal distribution model of vertebroplasty
}

\author{
Deguo Wang ${ }^{1,2}$, Yang $\mathrm{Li}^{2}$, Honglin $\mathrm{Yin}^{3}$, Jun $\mathrm{Li}^{2}$, Jiao $\mathrm{Qu}^{2}$, Minbo Jiang ${ }^{2}$, Jiwei Tian ${ }^{1}$ \\ ${ }^{1}$ Shanghai General Hospital of Nanjing Medical University, Shanghai 201600, China; ${ }^{2}$ Shanghai Songjiang District Central Hospital, Shanghai \\ 200090, China; 'School of Materials Science and Engineering, Shanghai Jiao Tong University, Shanghai 200240, China \\ Contributions: (I) Conception and design: D Wang; (II) Administrative support: J Tian; (III) Provision of study materials: H Yin, Y Li; (IV) Collection \\ and assembly of data: J Qu, Y Li; (V) Data analysis and interpretation: M Jiang; (VI) Manuscript writing: All authors; (VII) Final approval of \\ manuscript: All authors. \\ Correspondence to: Jiwei Tian. Shanghai General Hospital of Nanjing Medical University, Shanghai 201600, China. Email: atlantice0@126.com; Yang \\ Li. Shanghai Songiiang District Central Hospital, Shanghai 201600, China. Email: 226602370@qq.com.
}

Background: Establishment of a three-dimensional (3D) finite element model of osteoporosis, the simulation fluid was used to enter the vertebral body to study the stiffness recovery of injured vertebral body under different perfusion and distribution conditions, and the stress analysis of adjacent vertebral body after percutaneous vertebroplasty (PVP) was carried out.

Methods: A healthy male volunteer was selected. Computed tomography (CT) scanning was performed from T11 to L2. MIMICS 15.0 and ABAQUS 6.11 software was used to extract CT findings, and a vertebral model of osteoporotic fracture was established. The flow physical field and conduction and diffusion physical field were coupled to simulate the process and parts of the bone cement injection into the vertebral fracture model. The quantities of bone cement injected into the vertebral fracture model were 2,4 , and $6 \mathrm{~mL}$, respectively. The diffusion range of bone cement was simulated on the simulated image, and the postinjection model of bone cement was obtained. For the simulation of vertebral movement, vertical downward, forward, and backward pressure of $300 \mathrm{~N}$ was applied on the model's surface. The stress changes in the upper and lower vertebrae and diseased vertebrae were calculated under different conditions.

Results: It was revealed that the von Mises stress in the endplate under T12 was the highest in the three different states before and after fracture. The von Mises stress in the intervertebral discs and endplates was significantly higher after fracture than before fracture. When PVP was applied, the von Mises stress in adjacent endplates was increased with the increase of cement injection, while the von Mises stress was decreased in the adjacent endplates with cement injection compared with diseased vertebrae.

Conclusions: A reliable biomechanical model of lumbar vertebral fracture can be established through numerical simulation of CT scanning data. Vertebral fracture and vertebroplasty may cause biomechanical changes in adjacent vertebrae. The influence of biomechanical changes may notably increase along with the amount of bone cement injected. In this study, PVP revealed $4 \mathrm{~mL}$ to be the optimal amount for cement injection.

Keywords: Vertebroplasty; finite element analysis; bone cement; biomechanics

Submitted Mar 04, 2020. Accepted for publication May 07, 2020.

doi: 10.21037/apm-20-955

View this article at: http://dx.doi.org/10.21037/apm-20-955 


\section{Introduction}

Along with the significant increases in average life expectancy and older population growth in recent years, a rise in the number of patients treated for osteoporotic vertebral compression fractures (OVCF) has been observed (1). For these fractures, treatment such as traditional bed rest or external fixation of braces can easily cause the bone to further decalcify and loosen, forming a vicious circle. Osteoporosis and the poor general condition of patients also limit the possibility for open reduction and internal fixation with a pedicle screw (2) In recent years, new minimally invasive techniques, such as percutaneous vertebroplasty (PVP) and percutaneous kyphoplasty (PKP), have gradually been applied and demonstrated to have a marked effect, especially in the treatment of $\operatorname{OVCF}(3,4)$. However, as the application range and the number of follow-up cases have increased, a growing number of complications have been reported (5).

The major complications encountered in the treatment of OVCF can be short- or long-term. Intraoperative complications include toxic reactions to bone cement, damage to the spinal dura mater during puncture. Postoperative short-term complications mainly involve tamponade of the vertebral canal and nerve root compression caused by leakage of bone cement, while the fracture of adjacent segments is a common postoperative long-term complication (6). Uppin et al. (7), who performed follow-up of 177 patients with PVP over the course of more than 2 years, suggested that a new fracture was present in $12.4 \%$ of patients, $67 \%$ of whom reported the occurrence at the segments adjacent to the strengthened vertebrae. Secondary fractures of the adjacent segments after vertebroplasty are often the main complications influencing patients' postoperative long-term recovery and satisfaction. Therefore, a surgical technique that is relatively safe with few complications is urgently called for. Our experiment aimed to apply Navier Stokes equation of momentum conservation motion to describe the movement of bone cement, which is the first time to simulate the injection of bone cement into the vertebral body of osteoporotic fracture, and to inject the instantaneous incompressible viscous fluid bone cement into porous cancellous bone. The distribution of bone cement in the three-dimensional (3D) finite model is obtained. In order to study the best perfusion and distribution of the vertebral body in vertebroplasty, and the relationship between the distribution and the recovery of the stiffness of the diseased vertebral body and the increase of the stress of the adjacent vertebral body.

\section{Methods}

\section{Establishing the finite element model}

A 35-year-old healthy male free from other diseases was admitted to Central Hospital of Songjiang District (Shanghai, China). After his consent was obtained, GE 64-slice spiral computed tomography (CT) was used to scan the thoracolumbar segments. The scanning conditions were as follows: $140 \mathrm{kV}, 200 \mathrm{~mA}$, layer thickness of $0.625 \mathrm{~mm}$, and no interval. MIMICS software was used to perform bone separation by defining thresholds, forming masks, growing regions, editing, etc. T12-L2 bones were extracted, including $\mathrm{T} 12, \mathrm{~L} 1$, and L2 vertebrae along with $3 \mathrm{D}$ computer-aided design (CAD) models of the intervertebral discs. The material parameters of the bone were changed, to establish the osteoporosis model. The Remesh function was used to perform preliminary smoothing of the $3 \mathrm{D}$ model created using MIMICS software, and the Initial Graphics Exchange Specification (IGES) format file was output. Then, HyperMesh was imported to mesh, set boundary conditions and material parameters, and to perform other pretreatments. Finally, ABAQUS 6.11 software (ABAQUS Inc., Waltham, MA, USA) was utilized for computational solutions. To simulate the fracture, the height of the anterior edge of the L1 vertebral body was reduced by $10 \%$, and the elastic modulus of the cancellous bone was also decreased. Meanwhile, in line with previous studies (8-10), the respective characteristics of cortical and cancellous bone, the pedicle of the vertebral arch, transverse processes, spinous processes, intervertebral discs, and various ligaments of the vertebrae were defined (Tables 1 and 2). Eventually, the finite element model was created. Based on the osteoporotic fracture model, the simulation was performed for injecting the transient incompressible viscous flow fluid-bone cement into the cancellous bone (the momentum Navier-Stokes equation was used to describe the motion of the bone cement), and the process of injecting bone cement into a $3 \mathrm{D}$ vertebral body model was simulated to perform $3 \mathrm{D}$ distribution after the injection of bone cement into the vertebral body. The injection modes were 2, 4, and $6 \mathrm{~mL}$ on both sides, respectively. The diffusion range of the bone cement was outlined layer-by-layer on the simulated image, and the layers of each surgical vertebral body were calculated to obtain different bone cement perfusions and distribution 
Table 1 Material parameters of bone and ligaments

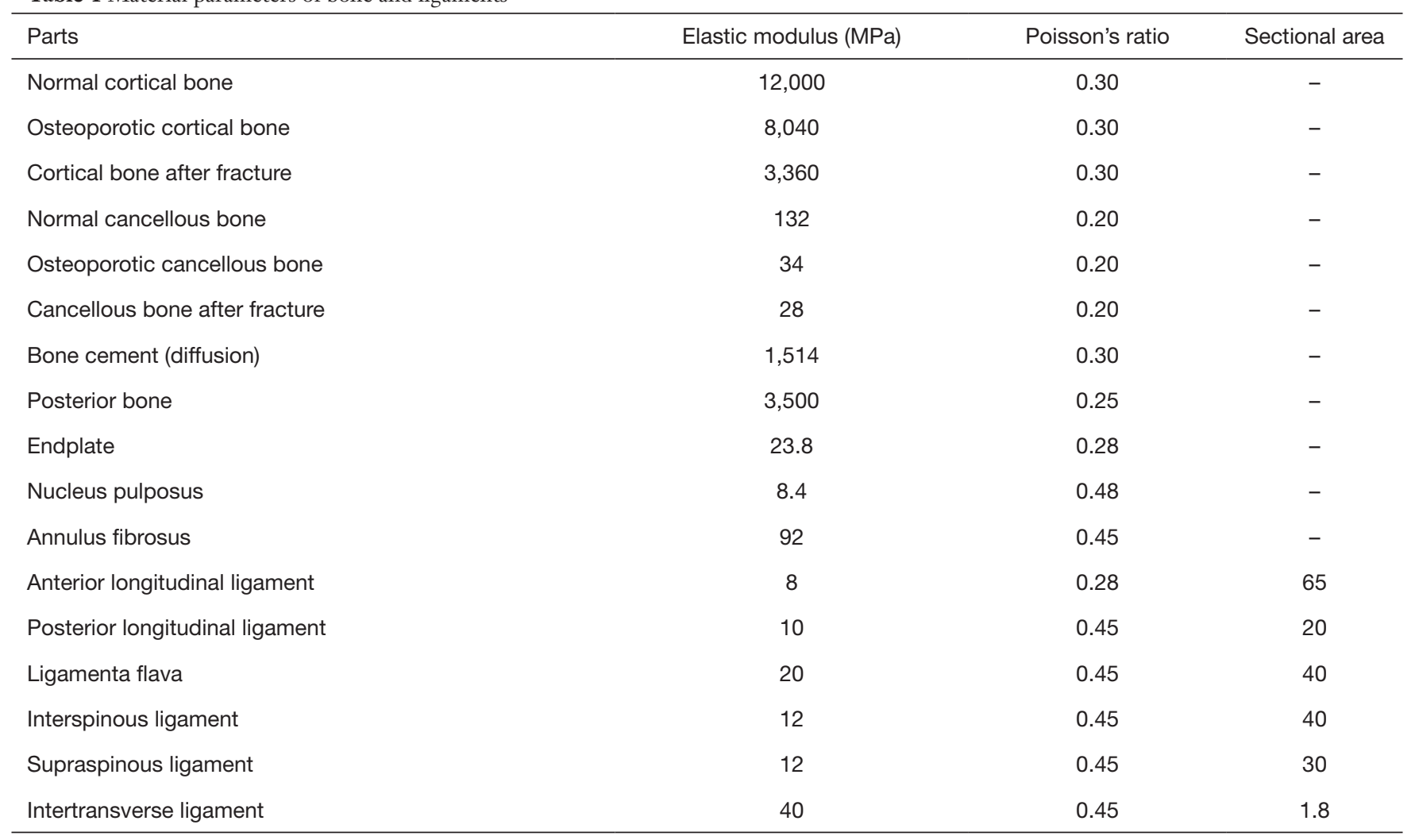

Table 2 Element type and material models

\begin{tabular}{|c|c|c|}
\hline Parts & Element type & Material model \\
\hline Cancellous bone & Tetrahedron & Linear elasticity \\
\hline Bone cement & Tetrahedron & Linear elasticity \\
\hline Endplate & Hexahedron & Linear elasticity \\
\hline Annulus fibrosus & Hexahedron & Linear elasticity \\
\hline Anterior longitudinal ligament & Truss element & Linear elasticity \\
\hline Posterior longitudinal ligament & Truss element & Linear elasticity \\
\hline Ligamenta flava & Truss element & Linear elasticity \\
\hline Intertransverse ligament & Truss element & Linear elasticity \\
\hline Capsular ligament & Truss element & Linear elasticity \\
\hline Capsular ligament & Truss element & Linear elasticity \\
\hline
\end{tabular}


patterns.

\section{Finite element model loading and analysis}

Three states were simulated in this experiment: standing, forward leaning, and backward extension. The loading conditions for standing were as follows: L2 bottom was fixed, and the axial compression load of $300 \mathrm{~N}$ (longitudinal along the $\mathrm{Z}$ axis) was applied in T12. In forward bending state, the axial compression load of $300 \mathrm{~N}$ and a $10 \mathrm{~nm}$ bending moment (along the $\mathrm{X}$-axis direction) were

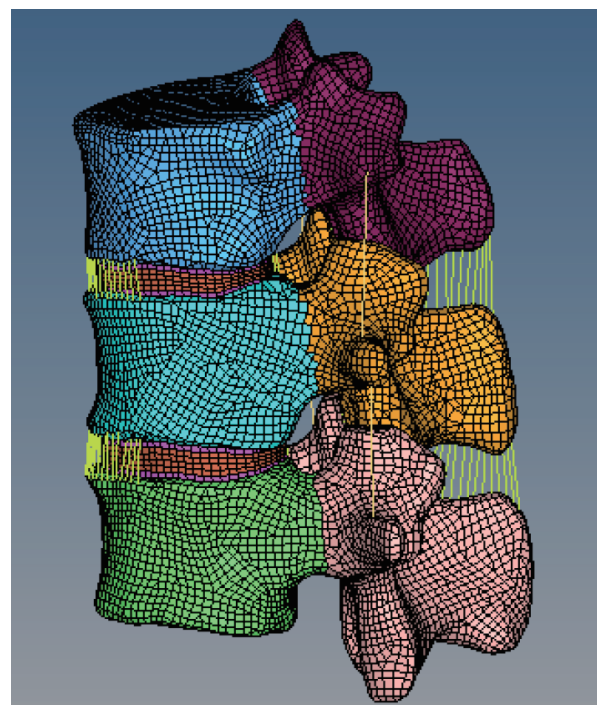

Figure 1 L1 osteoporotic fracture model after completion of assembly. applied in T12. In the backward extension state, the axial compression load of $300 \mathrm{~N}$ and a $10 \mathrm{~nm}$ bending moment (along the negative $\mathrm{X}$-axis direction) were applied in T12. The three states were applied to the osteoporotic fracture model, the 2-mL bone cement post-injection model, the 4-mL bone cement post-injection model, and the 6-mL bone cement post-injection model, respectively. The changes in stress distribution of the T12 vertebral inferior endplate, L1 vertebral superior and inferior endplates, and L2 vertebral superior endplate were calculated.

\section{Results}

Modeling: the T12-L2 model was created, from which the L1 vertebral model of osteoporotic fracture is shown in Figure 1

The model was established based on the anatomical data of different ligaments and bones. The anterior edge of the L1 vertebral body was reduced in height by $10 \%$, and the elastic modulus of cancellous bone was reduced by reference. The model was assembled to produce a realistic simulation of osteoporotic fracture, as shown in Figure 1.

\section{Simulation of fluid flow into the fractured vertebral body (Figure 2)}

Based on the osteoporotic fracture model, transient incompressible viscous fluid bone cement was injected into porous cancellous bone (the Navier-Stokes momentum conservation equation was applied to describe the flow of bone cement), and then, under general conditions, the

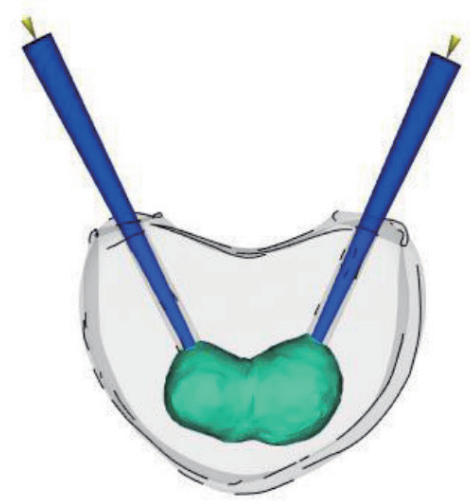

$2 \mathrm{~mL}$ bone cement

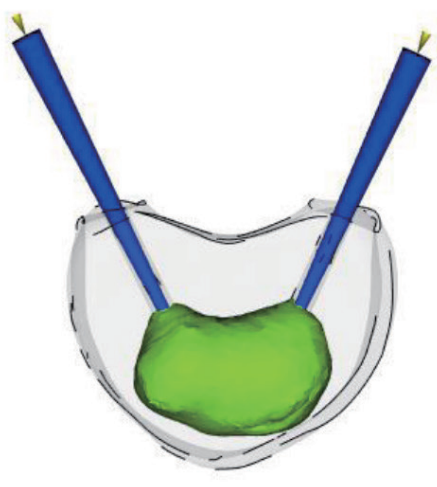

$4 \mathrm{~mL}$ bone cement

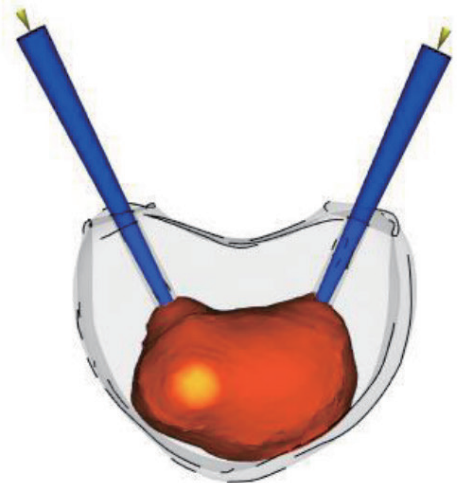

$6 \mathrm{~mL}$ bone cement

Figure 2 Simulation of the bone cement injection into the vertebral body, and the quantities of bone cement were 2, 4, and $6 \mathrm{~mL}$, respectively. The dark green stands for $2 \mathrm{~mL}$ bone cement. The light green stands for $4 \mathrm{~mL}$ bone cement. The red stands for $2 \mathrm{~mL}$ bone cement. 
process of injecting bone cement into the $3 \mathrm{D}$ vertebral model was simulated. Simultaneously, the 3D distribution of bone cement injected into the vertebral body was obtained. The injection patterns were: bilateral $2 \mathrm{ml}$, bilateral $4 \mathrm{ml}$, and bilateral $6 \mathrm{ml}$. The dispersion range of bone cement was sketched layer by layer on the simulated image. Every layer of each surgical vertebral body was calculated to determine the amount of bone cement perfusion and the distribution pattern, as shown in Figure 2.

\section{Results of pressure in the model in various states before and after simulated treatment}

Figure 3 shows the stress cloud pictures of the T12/L1 and L1/L2 intervertebral discs, T12 inferior endplate, L1 superior and inferior endplates, L2 superior endplate, and T12, L1, and L2 vertebrae under vertical compression before and after simulated treatment.

Figure 4 shows the stress cloud pictures of the T12/ L1, L1/L2 intervertebral discs, T12 inferior endplate, L1 superior and inferior endplates, L2 superior endplate, and T12, L1, L2 vertebrae in the forward bending state before and after simulated treatment.

Figure 5 shows the stress cloud pictures of the T12/ L1, L1/L2 intervertebral discs, T12 inferior endplate, L1 superior and inferior endplates, L2 superior endplate, and T12, L1, L2 vertebrae in the backward extension state before and after simulated treatment.

Tables 3, 4, and 5 show the maximum stress values of the intervertebral discs and each endplate in different states.

The above-mentioned results revealed the following outcomes: (I) the von Mises stress in the endplate under T12, in which the fracture easily occurred, was the highest in the three states before and after fracture; (II) the von Mises stress in the intervertebral discs and endplates was significantly higher after fracture than before fracture. When PVP was applied, the von Mises stress in the adjacent endplates increased along with the increase of cement injection, while the von Mises stress in the adjacent endplates decreased with the increase of cement injection compared with the diseased vertebrae. Thus, $4 \mathrm{~mL}$ was the optimal amount of injection of bone cement for the PVP in this experiment.

\section{Discussion}

The finite element method uses mathematical approximation to process a complex physical system through a number of small units, known as finite elements; thereby complex problems can be simplified. The FEM does not require several animal carcass specimens, meaning it can be applied for long-term repeated experiments, and therefore holds incomparable advantages in biomechanical simulation. Since the 1990s, finite element analysis has undergone rapid development in the field of spine surgery (8-11).

In recent years, PVP has been widely applied to treat osteoporotic fractures, owing to its minimal trauma, quick recovery time, and effectiveness. However, with the increasing duration of patient follow-up, related complications have gradually presented. Tanigawa et al. (12) performed follow-up for an average of 33 months in 194 patients, discovering that $33 \%$ of patients suffered fractures after PVP, and $67 \%$ of fractures occurred in the adjacent vertebrae, which was significantly higher than the rate in the non-adjacent segments. Secondary fractures of vertebral adjacent segments after vertebroplasty are often the main complications, negatively impacting the postoperative long-term efficacy of PVP and patient satisfaction. The reason may be attributed to the higher viscosity at which the bone cement is injected, which often restricts the diffusion distribution of the bone cement in the vertebral body. As osteoporosis causes post-fracture compression of the pore structure in areas of trabecular bone, only a certain space is left to accommodate the bone cement with high viscosity, thus limiting the distribution of the bone cement, and making the uniform distribution in the whole vertebral body unattainable. Furthermore, it destroys the initial pore and frame structures in the vertebral body, which are replaced with agglomerate bone cement, thereby changing the vertebral stiffness, elastic modulus, and other mechanical properties.

Generally, bone cement in a honeycomb shape with a wide distribution has a superior effect in recovering the mechanical properties of the whole vertebral body compared to bone cement with limited distribution. Polikeit et al. (13) demonstrated that even if a small amount of bone cement is injected, the stress and stress distribution of the adjacent segments can be significantly changed. This leads to an increase in the pressure on adjacent intervertebral discs and the deformation of the adjacent vertebral endplates, and causes the adjacent vertebrae to fracture, eventually resulting in the fracture of the entire vertebral body.

Baroud et al. $(8,14)$ used the finite element model to study the morphological changes of the endplates after vertebroplasty and stress conduction, and it was discovered 


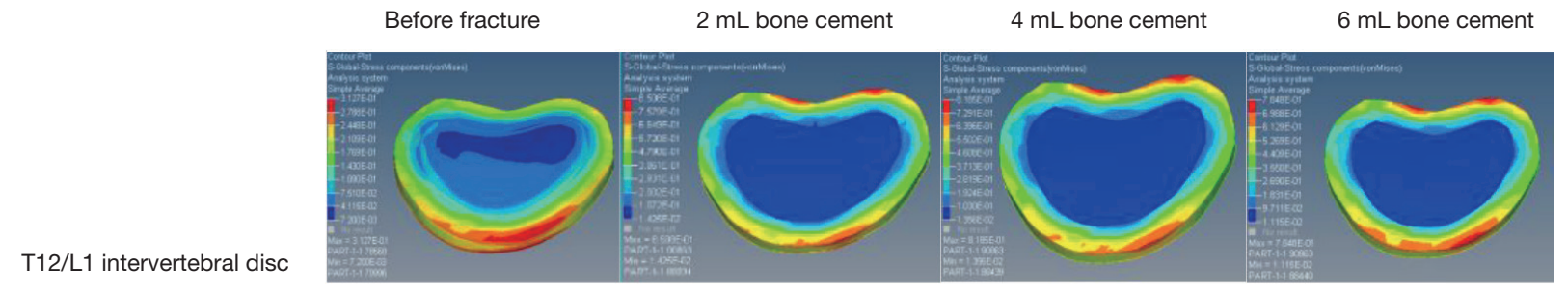

L1/2 intervertebral disc



T12 inferior endplate

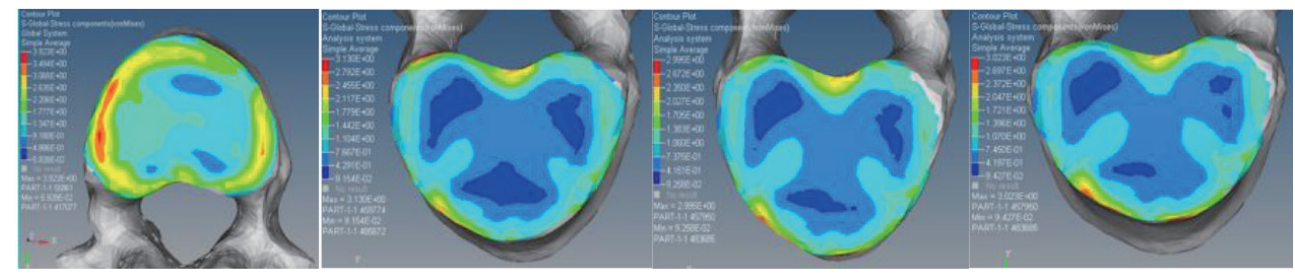

L2 superior endplate


L1 vertebra
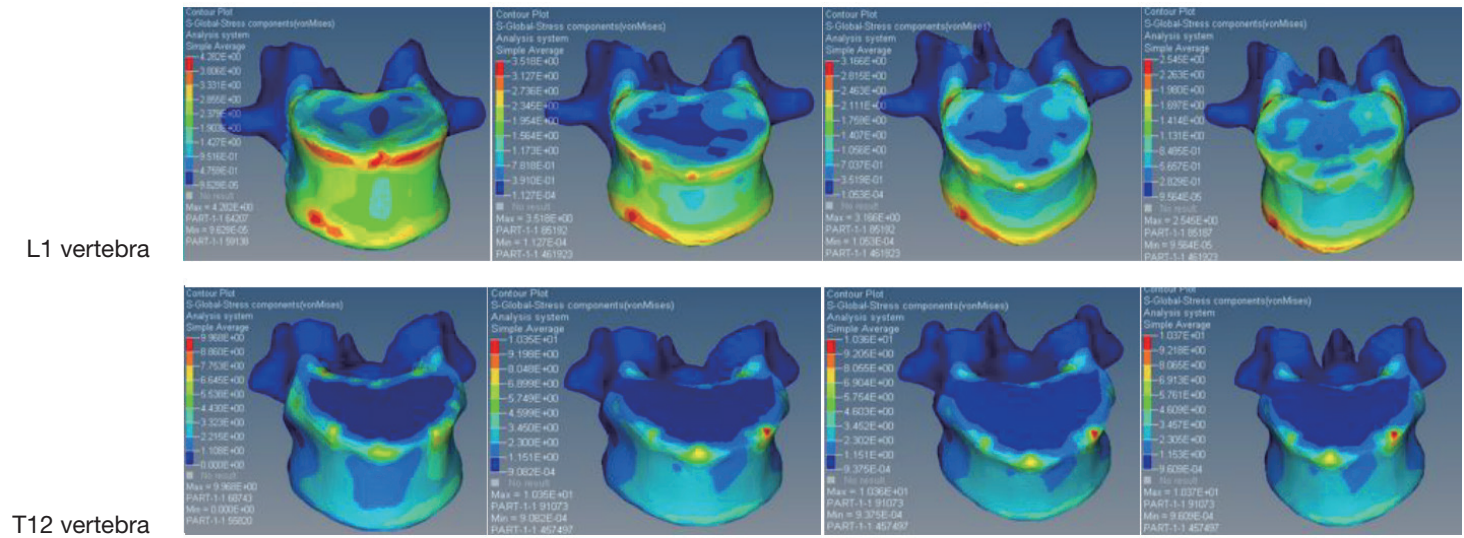

T12 vertebra
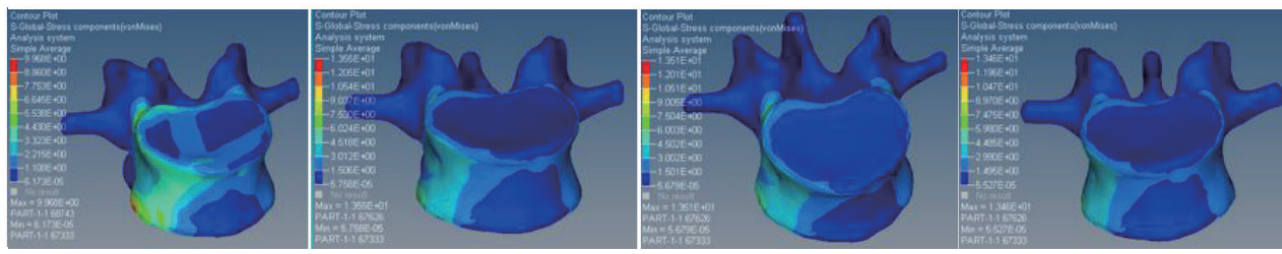

Figure 3 Stress cloud pictures of different parts under vertical compression. 




L1/2 intervertebral disc

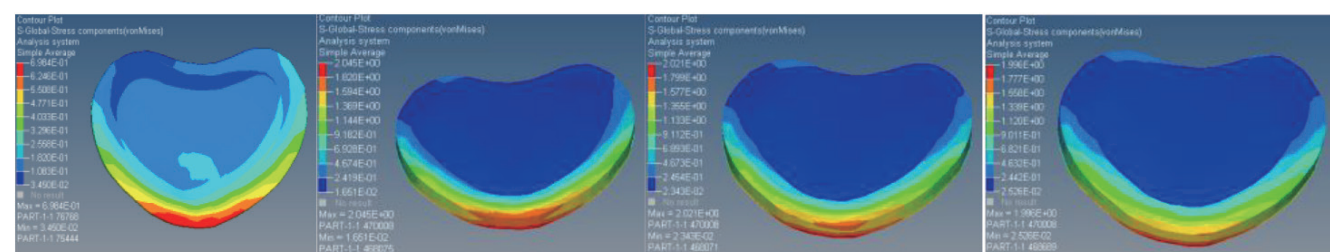

T12 inferior endplate
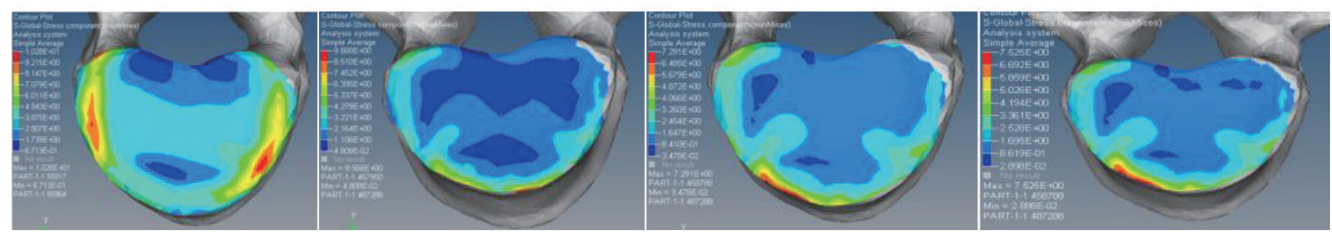

L2 superior endplate


L1 vertebra

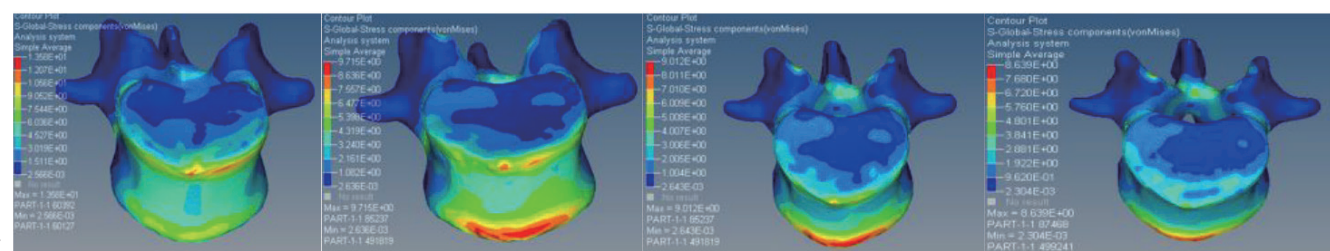

T12 vertebra

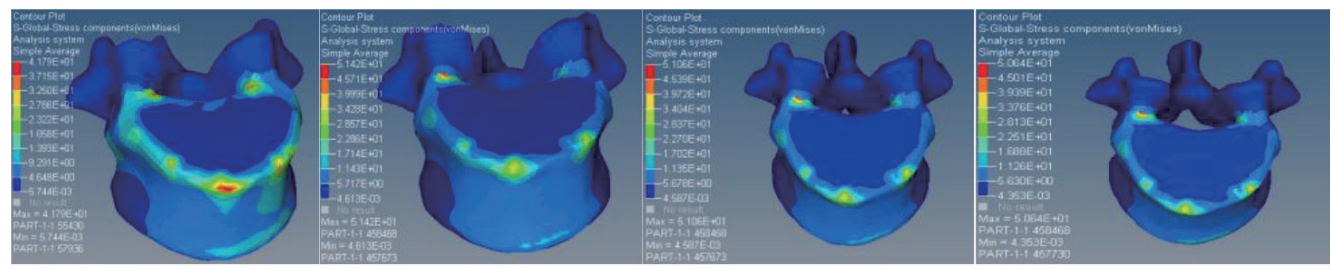

L2 vertebra

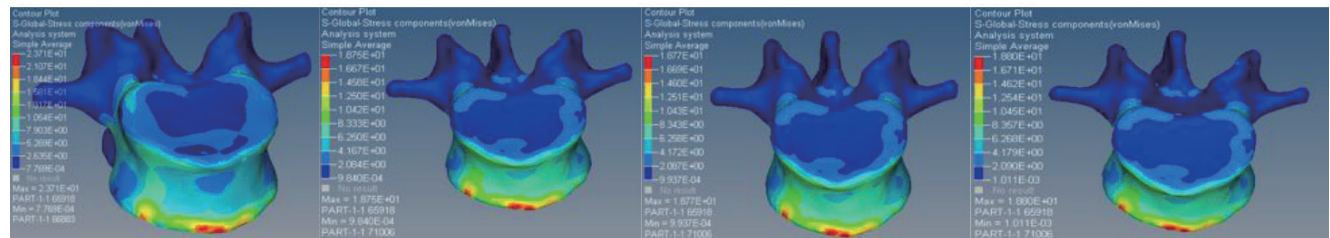

Figure 4 Stress cloud pictures of different parts in forward bending state. 

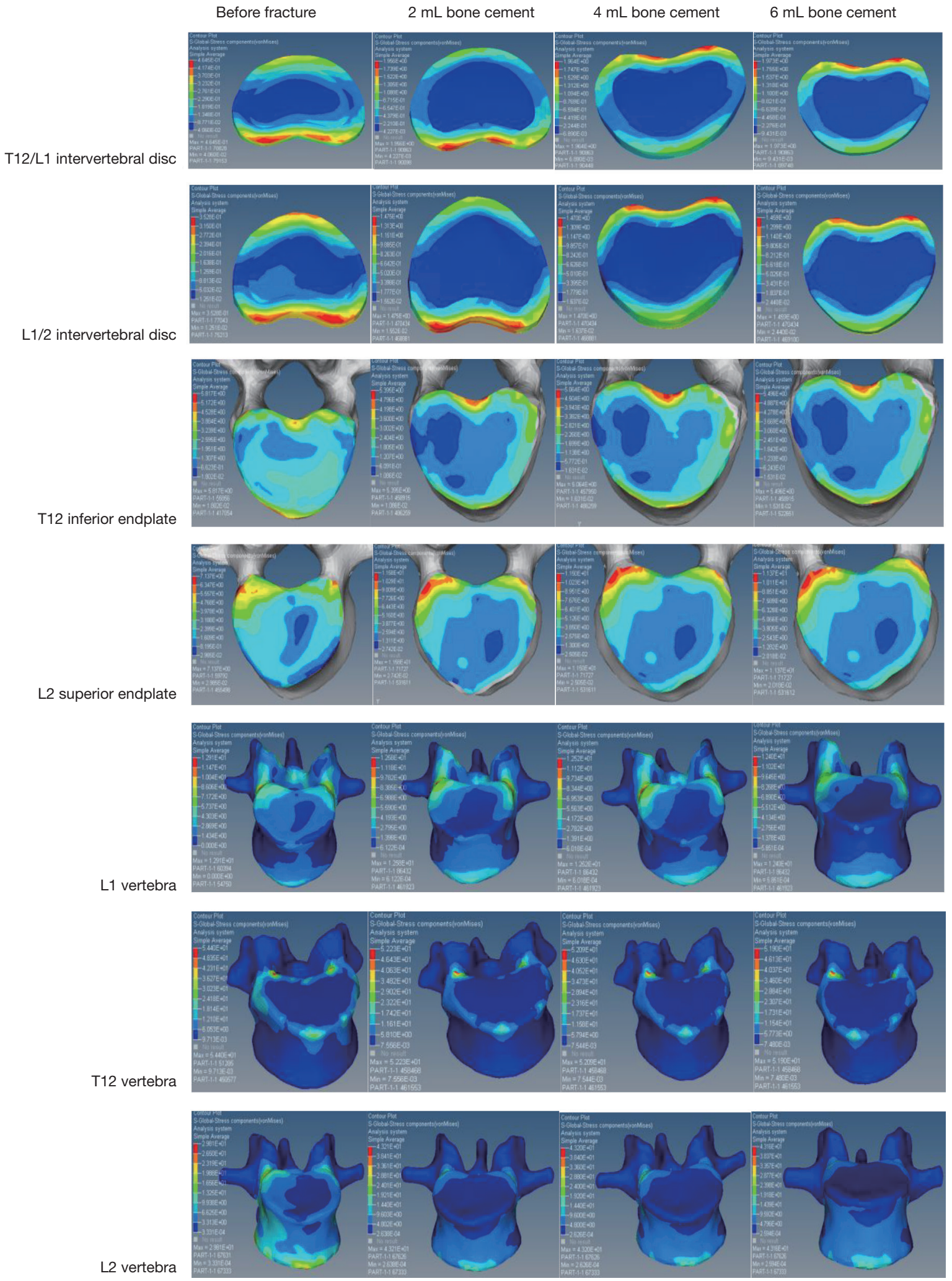

Figure 5 Stress cloud pictures of different parts in backward extension state. 
Table 3 Maximum stress values of intervertebral discs and each endplate under vertical compression (unit: MPa)

\begin{tabular}{|c|c|c|c|c|c|}
\hline Stress location & Before fracture & After fracture & Bone cement (2 mL) & Bone cement (4 mL) & Bone cement ( $6 \mathrm{~mL})$ \\
\hline L1/2 intervertebral disc & 0.2568 & 0.6735 & 0.6334 & 0.6167 & 0.6071 \\
\hline T12 inferior endplate & 0.6300 & 0.9750 & 0.9556 & 0.9319 & 0.9054 \\
\hline L1 superior endplate & 0.6377 & 0.8771 & 0.7237 & 0.667 & 0.6221 \\
\hline L2 superior endplate & 0.6136 & 0.7996 & 0.7929 & 0.7927 & 0.7968 \\
\hline
\end{tabular}

Table 4 Maximum stress values of intervertebral discs and each endplate in the forward bending state (unit: MPa)

\begin{tabular}{|c|c|c|c|c|c|}
\hline Stress location & Before fracture & After fracture & Bone cement (2 mL) & Bone cement (4 mL) & Bone cement ( $6 \mathrm{~mL})$ \\
\hline L1/2 intervertebral disc & 0.6984 & 2.129 & 2.045 & 2.021 & 1.996 \\
\hline T12 inferior endplate & 1.7450 & 2.528 & 2.594 & 2.634 & 2.716 \\
\hline L1 superior endplate & 1.7360 & 2.490 & 2.118 & 1.882 & 1.673 \\
\hline L2 superior endplate & 1.7700 & 2.377 & 2.380 & 2.379 & 2.382 \\
\hline
\end{tabular}

Table 5 Maximum stress values of intervertebral discs and each endplate in the backward extension state (unit: MPa)

\begin{tabular}{|c|c|c|c|c|c|}
\hline Stress location & Before fracture & After fracture & Bone cement (2 mL) & Bone cement (4 mL) & Bone cement (6 mL) \\
\hline L1/2 intervertebral disc & 0.3528 & 1.439 & 1.475 & 1.47 & 1.459 \\
\hline T12 inferior endplate & 0.9319 & 2.291 & 2.199 & 2.258 & 2.271 \\
\hline L1 superior endplate & 0.5712 & 1.534 & 1.481 & 1.535 & 1.538 \\
\hline L2 superior endplate & 0.5354 & 1.416 & 1.314 & 1.352 & 1.372 \\
\hline
\end{tabular}

that the rigid bone cement mass under the endplate played a vertical supporting role and reduced physiological recession of the endplates, increasing the internal pressure of the adjacent intervertebral discs by $19 \%$. In this case, the physiological recession of the endplates was taken as the cause of fracture in the adjacent vertebrae. A number of scholars also believe that the uneven distribution of bone cement in the vertebral body is typically the main cause of secondary fractures of adjacent vertebrae after vertebroplasty.

In this experiment, by using the CT scan data of 35 year old normal male, by changing the material parameters of bone, the $3 \mathrm{D}$ finite element model of osteoporosis is established, which can well simulate the fracture model in the state of osteoporosis (8-10). This study is the first to create a 3D finite element model of osteoporosis to inject the transient incompressible viscous flow fluid-bone cement into cancellous bone, and the process of injecting bone cement into a $3 \mathrm{D}$ vertebral model was simulated to obtain the $3 \mathrm{D}$ distribution of bone cement after injection into the vertebral body. In contrast with the previous methods of simply replacing the vertebral tissue with bone cement, 
this method was more effective in simulating the diffusion effect of bone cement in vertebral body, which more closely represented the distribution of bone cement after PVP. However, the study of the distribution of agglomerated or flat bone cement had some shortcomings, and this will be addressed in our future research.

The present research experiment studied the stiffness recovery of diseased vertebrae and the stress analysis of the adjacent vertebrae after simulating fluid flow into the vertebral body by injecting different amounts of bone cement. The Von Mises stress in the endplate under T12, in which fracture can easily occur, was revealed as the highest in the three states both before and after the fracture. This was also consistent with the rule of the multiple fracture sites and the pathogenesis of adjacent vertebrae fractures.

Simultaneously, we found that the Von Mises stress in the intervertebral discs and each endplate was significantly higher after fracture than before fracture. After PVP was applied, the Von Mises stress in the adjacent vertebral endplates increased along with the increase of bone cement injection. Berlemann et al. (15) pointed out, based on the finite element study, that the greater the amount of bone cement, the more easily the adjacent vertebrae would fracture. Compared with the diseased vertebra, the Von Mises stress in the vertebral endplate decreased with the increase of bone cement, which indicated that stress in the adjacent vertebral endplate significantly increased with a larger amount of bone cement, thus elevating the risk of fracture in the adjacent vertebrae. However, a small amount of bone cement cannot recover stiffness of the vertebral body: the smaller the amount of bone cement, the greater the Von Mises stress in the endplates of the diseased vertebrae. In this experiment, $4 \mathrm{~mL}$ was established as the optimal amount of bone cement for PVP, which can achieve appropriate clinical efficacy, while reducing the risk of secondary fractures of adjacent vertebrae and cement leakage caused by treatment with bone cement. This experiment successfully established the thoracolumbar spine model, which is not only suitable to study the biomechanical changes after the simulated bone cement injection, but also to study the biomechanical changes when different materials are applied to the spine. Lay the foundation for the development of new materials and technologies.

\section{Acknowledgments}

Funding: None.

\section{Footnote}

Data Sharing Statement: Available at http://dx.doi. org/10.21037/apm-20-955

Conflicts of Interest: All authors have completed the ICMJE uniform disclosure form (available at Available at http:// dx.doi.org/10.21037/apm-20-955). The authors have no conflicts of interest to declare.

Ethical Statement: The authors are accountable for all aspects of the work in ensuring that questions related to the accuracy or integrity of any part of the work are appropriately investigated and resolved. Informed consent was obtained from of the subjects who participated with the investigation.

Open Access Statement: This is an Open Access article distributed in accordance with the Creative Commons Attribution-NonCommercial-NoDerivs 4.0 International License (CC BY-NC-ND 4.0), which permits the noncommercial replication and distribution of the article with the strict proviso that no changes or edits are made and the original work is properly cited (including links to both the formal publication through the relevant DOI and the license). See: https://creativecommons.org/licenses/by-nc-nd/4.0/.

\section{References}

1. Cheng X, Long HQ, Xu JH, et al. Comparison of unilateral versus bilateral percutaneous kyphoplasty for the treatment of patients with osteoporosis vertebral compression fracture (OVCF): a systematic review and meta-analysis. Eur Spine J 2016;25:3439-49.

2. Wang XR, Kwok TC, Griffith JF, et al. Prevalence of cervical spine degenerative changes in elderly population and its weak association with aging, neck pain, and osteoporosis. Ann Transl Med 2019;7:486.

3. De Negri P, Tirri T, Paternoster G, et al. Treatment of painful osteoporotic or traumatic vertebral compression fractures by percutaneous vertebral augmentation procedures: a nonrandomized comparison between vertebroplasty and kyphoplasty. Clin J Pain 2007;23:425-30.

4. Burton AW, Hamid B. Kyphoplasty and vertebroplasty. Curr Pain Headache Rep 2008;12:22-7.

5. Wang H, Sribastav SS, Ye F, et al. Comparison of Percutaneous Vertebroplasty and Balloon Kyphoplasty 
for the Treatment of Single Level Vertebral Compression Fractures: A Meta-analysis of the Literature. Pain Physician 2015;18:209-22.

6. Zhang L, Li J, Yang H, Luo Z, Zou J. Histological evaluation of bone biopsy results during PVP or PKP of vertebral compression fractures. Oncol Lett 2013;5:135-8.

7. Uppin AA, Hirsch JA, Centenera LV, et al. Occurrence of new vertebral body fracture after percutaneous vertebroplasty in patients with osteoporosis. Radiology. 2003;226:119-24.

8. Boger A, Heini P, Windolf M, et al. Adjacent vertebral failure after vertebroplasty: a biomechanical study of lowmodulus PMMA cement. Eur Spine J 2007;16:2118-25.

9. Han KS, Rohlmann A, Yang SJ, et al. Spinal muscles can create compressive follower loads in the lumbar spine in a neutral standing posture. Med Eng Phys 2011;33:472-8.

10. Liebschner MA, Kopperdahl DL, Rosenberg WS, et al. Finite element modeling of the human thoracolumbar

Cite this article as: Wang D, Li Y, Yin H, Li J, Qu J, Jiang M, Tian J. Three-dimensional finite element analysis of optimal distribution model of vertebroplasty. Ann Palliat Med 2020;9(3):1062-1072. doi: 10.21037/apm-20-955 spine. Spine (Phila Pa 1976) 2003;28:559-65.

11. Pintar FA, Yoganandan N, Myers T, et al. Biomechanical properties of human lumbar spine ligaments. J Biomech 1992;25:1351-6.

12. Tanigawa N, Kariya S, Komemushi A, et al. Percutaneous vertebroplasty for osteoporotic compression fractures: long-term evaluation of the technical and clinical outcomes. AJR Am J Roentgenol 2011;196:1415-8.

13. Polikeit A, Nolte LP, Ferguson SJ. The effect of cement augmentation on the load transfer in an osteoporotic functional spinal unit: finite-element analysis. Spine (Phila Pa 1976) 2003;28:991-6.

14. Baroud G, Nemes J, Heini P, et al. Load shift of the intervertebral disc after a vertebroplasty: a finite-element study. Eur Spine J 2003;12:421-6.

15. Berlemann U, Ferguson SJ, Nolte LP, et al. Adjacent vertebral failure after vertebroplasty. A biomechanical investigation. J Bone Joint Surg Br 2002;84:748-52. 